cer, and often alterations occur through mutations in p16 and the human homolog of p19 (p14). So it's not surprising that INK4a/ARF-knockout mice are prone to cancer. They develop mainly hematopoietic tumors, some melanomas and fibrosarcomas and, rarely, rhabdomyosarcomas. The tumors in either $H G F / S F$-transgenic or INK4a/ARF-knockout mice resemble those that develop in adult humans more than those in children, and both strains have a relatively long average lifespan over a 16-month interval.

The surprise came from mice that expressed the $H G F / S F$ transgene and, in addition, were deficient for INK4a/ARF. The doubly mutant mice developed rhabdomyosarcomas with nearly a $100 \%$ frequency and died rapidly within 4-5 months. The mouse tumors had several features in common with their human counterparts: some muscle striations, stabilized p53 and expression of desmin, $\alpha$-actin, MYOD1 and Pax7. Moreover, myoblast precursor cells appeared to differentiate poorly, suggestive of the arrested or abortive differentiation of human rhabdomyosarcomas: While proliferating well in culture, these precursors were unable to fuse to form myotubes. Mice with this genetic combination represent the most faithful and predictable animal model of rhabdomyosarcoma yet.

The precise mechanisms by which this particular combination of mutations causes the selective and unusual development of rhabdomyosarcomas are unclear. However, it is tempting to speculate that it is the consequence of dysregulated proliferation and survival of rhabdomyoblasts coupled to a loss of orderly cell-cycle control. This combination could have the effect of allowing precursor cells to proliferate for a longer period of time before terminal differentiation, thereby increasing the target pool for transforming events. Because not all rhabdomyoblasts in these mice transform, it is clear that other mutations layered onto this background drive tumor progression. This raises several questions.

Would any proliferation advantage coupled to a cell-cycle defect lead to the development of rhabdomyosarcomas? Perhaps it is the combination of specific deregulated signaling and the rhabdomyoblast cell milieu which elicits the tumor type. The early tumor development typical of childhood cancer may also result from the coupling of specific deregulating mutations in early stages of tissue development. It will also be important to determine whether other genetic lesions seen in these tumors are actually secondary in human rhabdomyosarcoma or if they can be primary initiators under some circumstances. It is possible that different combinations of mutations give rise to the several histological and genetic subtypes of rhabdomyosarcoma in humans. Finally, the stage of muscle development at which transformation occurs might affect phenotype, tumor location or tumor aggressiveness.

Testing of preclinical candidate therapies should now be enhanced for these tumors. Perhaps most interesting is the ability to directly dissect the relationship of muscle development and tumorigenesis. It may be asking too much of the present set of mouse strains to answer the numerous outstanding questions about rhabdomyosarcoma and related cancers. But clearly, the work has just begun.

1. Enzinger, F.M. \& Weiss, S.W. Soft Tissue Tumors 3rd edn. (C.V. Mosby, St. Louis, 2000).

2. Sharp, R. et al. Synergism between INK4a/ARF inactivation and aberrant HGF/SF signaling in rhabdomyosarcomagenesis. Nature Med. 8 1276-1280 (2002).

3. Tonin, P.T. et al. Muscle-specific gene expression in rhabdomyosarcomas and stages of human fetal skeletal muscle development. Cancer Res. 51, 5100-5106 (1991)

4. Maelandsmo, G.M. et al. Homozygous deletion frequency and expression levels of the CDKN2A gene in human sarcomas-relationship to amplification and mRNA levels. Br. J. Cancer 72, 393-398 (1995).

5. Mulligan, L.M. et al. Mechanisms of p53 loss in human sarcomas. Proc. Natl. Acad. Sci. USA 87, 5863-5867 (1990).

6. Merlino, G. \& Helman, L.J. Rhabdomyosarcomaworking out the pathways. Oncogene 12, 1697-1705 (1996).

7. Ferracini, R. et al. Retrogenic expression of the MET proto-oncogene correlates with the invasive phenotype of human rhabdomyosarcoma. Oncogene 12, 1697-1705 (1996).

8. Takayama, H. et al. Diverse tumorigenesis associated with aberrant development in mice overexpressing hepatocyte growth factor/scatter factor. Proc. Natl. Acad. Sci. USA 94, 701-706 (1997).

9. Serrano, M. et al. Role of the INK4a locus in tumo suppression and cell mortality. Cell 85, 27-37 (1996).

Ludwig Institute for Cancer Research Department of Medicine, Cancer Center and Center for Molecular Genetics

University of California at San Diego

La Jolla, California, USA

Email:wcavenee@ucsd.edu

\title{
Two-sided T cell
}

A T cell becomes activated when it encounters an antigen presented on a $B$ cell or other antigen-presenting cell (APC). The resulting changes in gene expression drive T-cell proliferation, differentiation and effector functions. In the November 1 Nature Immunology, Costello et al. take a close look at the signaling that leads to these events. A key molecule in this process is phosphatidylinositol $(3,4,5)$ triphosphate $\left(\mathrm{PIP}_{3}\right)$. This lipid binds to a pleckstrin homology domain in a diverse array of signal transducing proteins, controlling their activity and subcellular localization. To visualize $\mathrm{PIP}_{3}$ activity, the authors used a previously designed fusion protein consisting of this domain fused to green fluorescent protein (GFP). In this image, high-intensity GFP fluorescence is red, medium is yellow and low intensities are green and blue. Within a minute of APC-antigen contact, the GFP fusion protein relocated from the cytoplasm to the cell membrane near the APC (APC on left). Shortly thereafter, most T cells also

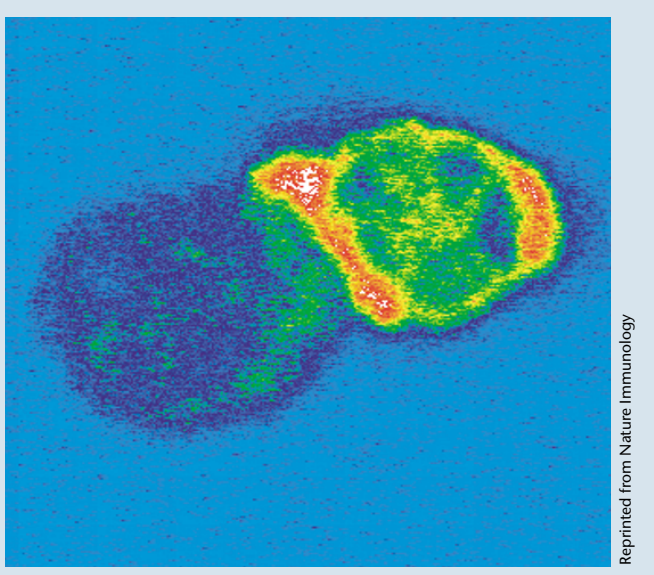
developed a second region of high PIP $_{3}$ activity at the opposite pole of the cell. The authors found that sustained PIP ${ }_{3}$ activity occurred for at least nine hours after APC contact and was essential for T-cell proliferation.

CharlotTe SCHUberT 